Trauma Berufskrankh $2006 \cdot 8$ [Suppl 2]:

S225-S228

DOI 10.1007/s10039-005-1088-5

Online publiziert: 31. Januar 2006

(c) Springer Medizin Verlag 2006
E. Scola

Klinikum Landkreis Neumarkt i.d.OPf, Neumarkt i.d.OPf.

\section{Diagnostik und Therapie der Algodystrophie}

\begin{tabular}{|c|c|c|c|c|c|}
\hline & Einheit & A. femoralis & $\begin{array}{l}\text { Linke V. cubitalis } \\
\text { (normal) }\end{array}$ & $\begin{array}{l}\text { Rechte V. cubitalis } \\
\text { (LIRS) }\end{array}$ & $\begin{array}{l}\text { Differenz links/ } \\
\text { rechts } \mathrm{pO}_{2} \text { venös }\end{array}$ \\
\hline \multicolumn{6}{|c|}{ Therapiebeginn } \\
\hline \multirow[t]{2}{*}{$\mathrm{pO}_{2}$} & {$[\mathrm{~Pa}]$} & 11.970 & 2660 & 5320 & 2660 \\
\hline & [Torr] & 90 & 20 & 40 & 20 \\
\hline \multirow[t]{2}{*}{$\mathrm{pCO}_{2}$} & {$[\mathrm{~Pa}]$} & 6650 & 8246 & 7182 & \\
\hline & [Torr] & 50 & 62 & 54 & \\
\hline \multicolumn{6}{|c|}{ Nach 1 Woche } \\
\hline \multirow[t]{2}{*}{$\mathrm{pO}_{2}$} & {$[\mathrm{~Pa}]$} & 11.704 & 4522 & 6251 & 1729 \\
\hline & [Torr] & 88 & 34 & 47 & 13 \\
\hline \multirow[t]{2}{*}{$\mathrm{pCO}_{2}$} & {$[\mathrm{~Pa}]$} & 5586 & 7581 & 6916 & \\
\hline & [Torr] & 42 & 57 & 52 & \\
\hline \multicolumn{6}{|c|}{ Nach 2 Wochen } \\
\hline \multirow[t]{2}{*}{$\mathrm{pO}_{2}$} & {$[\mathrm{~Pa}]$} & 11.970 & 2926 & 4389 & 1463 \\
\hline & [Torr] & 90 & 22 & 33 & 11 \\
\hline \multirow[t]{2}{*}{$\mathrm{pCO}_{2}$} & {$[\mathrm{~Pa}]$} & 6251 & 7182 & 6783 & \\
\hline & [Torr] & 47 & 54 & 51 & \\
\hline
\end{tabular}

Jegliche Art von Gewebeschaden geht mit einer Freisetzung von Entzündungsmediatoren einher. In Abhängigkeit vom Ausmaß des Defekts und der Membranstabilität der Zellen ist die Entzündungsreaktion unterschiedlich stark. Durch lokal begrenzte Entzündungskreisläufe kann gerade im Bereich ausgeprägter Mikrozirkulation eine Minderperfusion durch Entwicklung von arterio-venösen Shunts entstehen, obwohl eine Hyperthermie und Rötung klinisch eine Hyperperfusion vortäuschen.

In Anlehnung an ein „systemic inflammatory response syndrome" (SIRS) wird im Stadium I der posttraumatischen Algodystrophie mit den bekannten Zeichen der Entzündung eine lokale Entzündungsreaktion (LIRS) mit der Ausbildung von arterio-venösen Shunts diskutiert [1, 3, 4, 5, 6]. Ziel der Behandlung sollte daher sein, die Rheologie im Bereich der Kapillaren so zu verbessern, dass diese Shunts vermindert werden.

\section{Material und Methode}

In den Jahren von 2000-2005 wurden 5 Patienten mit distaler Radiusfraktur mit Verdacht auf Algodystrophie im Stadium I behandelt (4 C-Frakturen, 1 B-Fraktur). Alle Patienten wurden nach dem gleichen Schema therapiert, das exemplarisch an einem Fallbeispiel erläutert wird.

\section{Fallbeispiel}

Ein 52-jähriger Betonbauer zog sich eine distale intraartikuläre Radiustrümmer-
Tab. 2 Therapiekonzept bei arterio-venösem Shunt

\begin{tabular}{|c|c|c|}
\hline \multicolumn{2}{|l|}{ Maßnahme } & \multirow{2}{*}{$\begin{array}{l}\text { Dosierung/Menge } \\
\text { 3-mal } 250 \mathrm{ml} \mathrm{i.v.}\end{array}$} \\
\hline Rheologika & Haes 6\% & \\
\hline Antioxidanzien & Ascorbinsäure & 1000 mg tgl. \\
\hline Antiphlogistika & Diclofenac retard & $1 / 0 / 1$ \\
\hline Analgetika & Tramadol $100 \mathrm{mg}$ & $1 / 0 / 1$ \\
\hline Magenschutz & Omeprazol $20 \mathrm{mg}$ & 1-mal tgl. \\
\hline \multirow{2}{*}{$\begin{array}{l}\text { Weitere Medi- } \\
\text { kation }\end{array}$} & Vitamin-B-Komplex & 3-mal 1 \\
\hline & Magnesium & 3-mal $250 \mathrm{mg}$ \\
\hline \multirow{5}{*}{$\begin{array}{l}\text { Balneo-Physio- } \\
\text { therapie }\end{array}$} & $\mathrm{CO}_{2}$-Bäder & 2-mal tgl. an beiden oberen Extremitäten \\
\hline & Lymphdränagen & 2-mal tgl. \\
\hline & $\begin{array}{l}\text { Aktive und passive Krankengym- } \\
\text { nastik mit Muskelaufbautraining }\end{array}$ & \\
\hline & Konsensuelle Therapie & 2-mal tgl. \\
\hline & lontophorese & \\
\hline \multirow[t]{3}{*}{$\begin{array}{l}\text { Lokale Anwen- } \\
\text { dungen }\end{array}$} & $\begin{array}{l}\text { Okklusivverbände zur Nacht mit } \\
\text { Antiphlogistikasalbe }\end{array}$ & \\
\hline & Hochlagerung & \\
\hline & Kühlmanschette & \\
\hline
\end{tabular}




\section{Körperferner Speichenbruch}
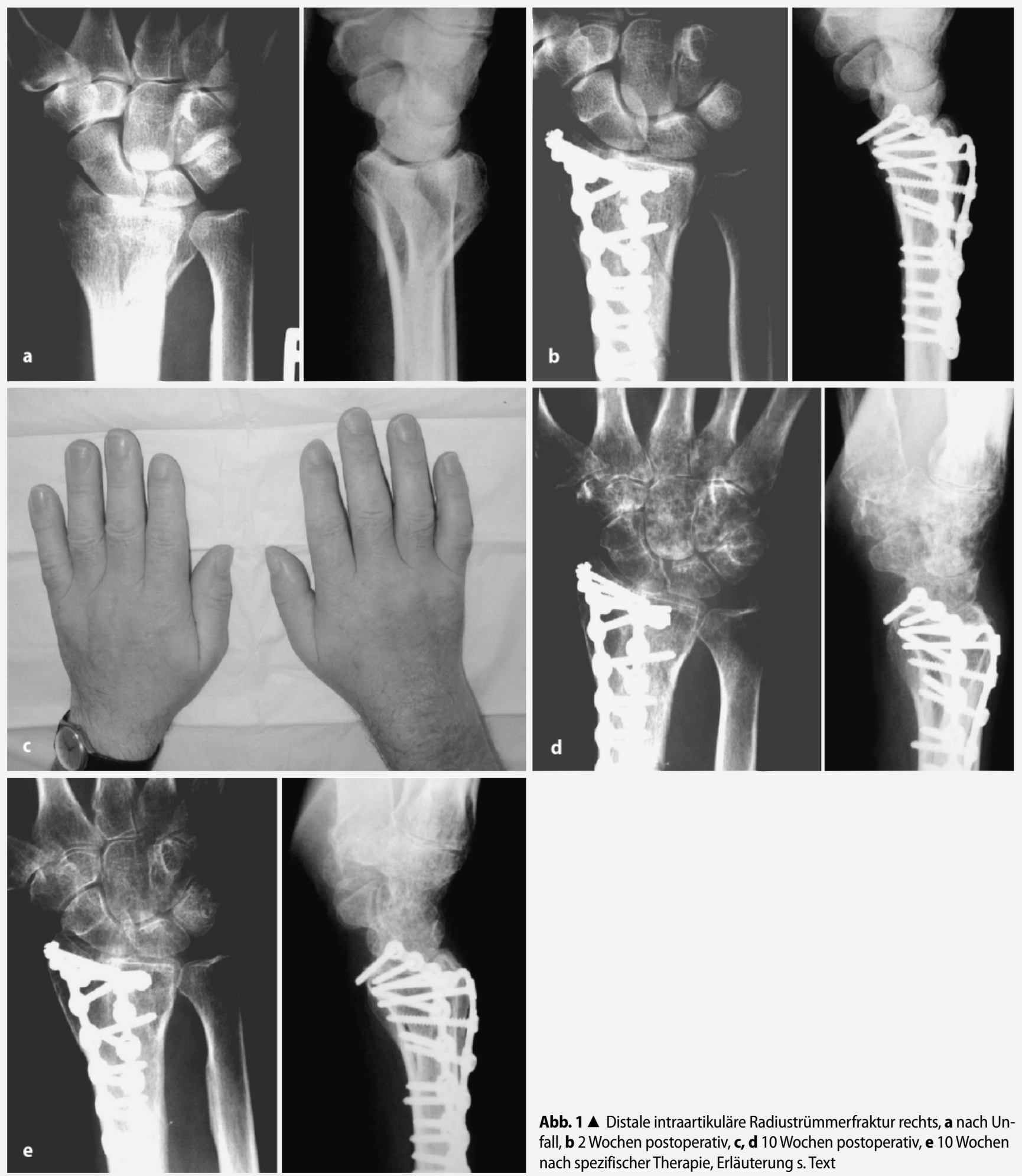

Abb. $1 \Delta$ Distale intraartikuläre Radiustrümmerfraktur rechts, a nach Unfall, b 2 Wochen postoperativ, c, d 10 Wochen postoperativ, e 10 Wochen nach spezifischer Therapie, Erläuterung s. Text

fraktur rechts (Typ $23 \mathrm{C}_{3.2}$ nach $\mathrm{AO}-\mathrm{Klas}$ sifikation) zu ( $\bullet$ Abb. 1a). Die primäre Versorgung wurde mit Fixateur externe und Spickdrähten vorgenommen. Wegen unzureichender Stellung der Gelenkfragmente erfolgte 8 Tage später ein Verfahrenswechsel (offene Reposition und $\mathrm{Pi}$ -
Platten-Osteosynthese) über einen dorsalen Zugang (• Abb. 1b).

Im Rahmen der ambulanten Nachbehandlung fielen nach 4 Wochen persistierende Schmerzen, Schwellung, Rötung, vermehrte Haarbildung und deutliche Bewegungseinschränkung auf (Stadium I der Algodystrophie). Es erfolgten Ruhigstel- lung, Gabe von Antiphlogistika und Analgetika. Nach weiteren 4 Wochen entwickelte sich eine zunehmend schmerzhafte Bewegungseinschränkung mit Schwellung und Muskeldystrophie (• Abb. 1c), radiologisch konnte eine typische fleckige Knochendystrophie nachgewiesen werden (• Abb. 1d) (beginnendes Stadium 
II der Algodystrophie). Zu diesem Zeitpunkt erfolgte die Blutgasanalyse aus beiden Ellenbeugen venös und aus der Leiste arteriell unter der Annahme von arterio-venösen Shunts ( $\bullet$ Tab. 1). Im venösen Blut der betroffenen rechten Extremität wurde im Vergleich zur linken Seite ein vermehrter $\mathrm{O}_{2}$-Gehalt gefunden. Das in - Tab. 2 dargestellte Therapiekonzept kam daraufhin zur Anwendung.

\section{Ergebnisse}

Innerhalb von 2 Wochen konnte eine bessere periphere Sauerstoffnutzung für die betroffene Extremität verzeichnet werden (Wochenkontrollen, - Tab. 1). Klinisch kam es zum Rückgang der Schwellung, Verbesserung der Beweglichkeit, Schmerzlinderung und radiologisch beginnender Rückbildung der fleckigen Knochendystrophie. 3 Monate später bestanden nahezu vollständiger Faustschluss, intakter Fingerspitzengriff, kein Ruheschmerz, normale Trophik und radiologisch normaler Kalksalzgehalt (• Abb. 1e).

\section{Schlussfolgerung}

Im Verlauf einer posttraumatischen Algodystrophie lässt sich ein LIRS auch blutgasanalytisch annehmen. Balneo-physikalische und medikamentöse Maßnahmen unter diesem Aspekt zeigen klinisch deutlich positive Effekte. Die venöse $\mathrm{pO}_{2}$ Differenz zwischen betroffener und normaler Extremität scheint mit dem Therapieerfolg gut zu korrelieren.

\section{Fazit für die Praxis}

Nach distaler Radiusfraktur und klinischen Zeichen einer beginnenden Algodystrophie, u. a. mit persistierenden Schmerzen, Schwellung, Rötung und Bewegungseinschränkung, sollte eine vergleichende venöse Blutgasanalyse zwischen dem verletzten und dem unverletzten Arm erfolgen. Zeigt sich dabei auf der verletzten Seite ein erhöhter $\mathrm{pO}_{2-}$ Wert, müssen arterio-venöse Shunts im präkapillaren Bereich angenommen werden. Dieser Zustand bewirkt auf Dauer eine Gewebedystrophie mit Dauerschmerzen (Algodystrophie). Um die Rheologie im kapillaren Bereich zu verbessern, bietet sich die Blockade der Ad-

Trauma Berufskrankh 2005 - 8[Suppl 2]: S225-S228 DOI 10.1007/s10039-005-1088-5

(c) Springer Medizin Verlag 2006

E. Scola

\section{Diagnostik und Therapie der Algodystrophie}

\section{Zusammenfassung}

In Anlehnung an ein posttraumatisches "Systemic inflammatory response syndrome" (SIRS) kann bei der posttraumatischen Algodystrophie eine lokale Entzündungsreaktion (LIRS) mit Ausbildung von arterio-venösen Shunts diskutiert werden. Werden nach einer distalen Radiusfraktur Zeichen eines Stadiums I der Algodystrophie entdeckt, sind eine venöse Blutgasanalyse aus der V. cubitalis des betroffenen und des unverletzten Arms indiziert. Bei erhöhtem venösem Sauerstoffgehalt kann durch Gabe von Rheologika, Analgetika, Antioxidanzien und Antiphlogistika zusammen mit balneo-physikalischen Maßnahmen versucht werden, den arterio-venösen Shunt zu drosseln. Bei 5 Patienten mit distaler Radiusfraktur und Stadium I der AI-

\section{Diagnosis and therapy of algodystrophy}

\section{Abstract}

Based on post-traumatic"systemic inflammatory response syndrome (SIRS)", a posttraumatic algodystrophy can be considered as a "lokal inflammatory response syndrome (LIRS)" with the appearance of arteriovenous shunts. If a stadium I algodystrophy occurs after distal radius fracture, venous blood gas analysis of the traumatized and non-traumatized arm are performed (cubital vein). In case of an elevated venous oxygen partial pressure in the affected arm, rheologic, analgesic, antioxidant and antiphlogistic drugs are applied and balneo-physical therapy performed to reduce the arteriovenous shunts. godystrophie wurde frühzeitig blutgasanalytisch ein arterio-venöser Shunt nachgewiesen. Nach 3-wöchiger Therapie konnten alle Patienten ambulant weiterbehandelt werden. Somit hat eine spezifische medikamentöse Therapie zusammen mit balneo-physikalischen Maßnahmen bei Patienten mit posttraumatischer Algodystrophie, bei denen blutgasanalytisch ein arterio-venöser Shunt nachgewiesen wurde, deutlich positive Effekte.

\section{Schlüsselwörter}

Algodystrophie · Radiusfaktur · Arterio-venöser Shunt · Entzündungsreaktion · Balneophysikalische Therapie
In five patients, we found that the levels of arteriovenous shunts decreased with this treatment. After 3 weeks, all patients were discharged to outpatient clinics. On the occurrence of evident post-traumatic algodystrophy, specific medical therapy to decrease arteriovenous shunts together with balneophysical therapy has a positive clinical effect.

\section{Keywords}

Algodystrophy - Radius fracture · Arterial-venous shunt $\cdot$ Inflammation $\cdot$ Balneo-physical therapy 


\section{Körperferner Speichenbruch}

häsine von korpuskulären Blutbestandteilen und Zelloberfläche durch Haes $6 \%$ an [2]. Zusätzlich sind analgetische Maßnahmen, die Gabe von Antiphlogistika und Antioxidanzien, kombiniert mit balneo-physikalischen Maßnahmen, zu empfehlen.

\section{Korrespondierender Autor}

Prof. Dr. E. Scola

Klinikum Landkreis Neumarkt i.d.OPf

Nürnbergerstraße 12, 92318 Neumarkt i.d.OPf.

Prof.Scola@Klinikum.Neumarkt.de

\section{Literatur}

1. Goris R, Jan A, Laan L van der (2001) Reflex sympathetic dystrophy - another view. Eur J Trauma 27: 99-103

2. Laubenthal H, Sirtl C (1998) Hydroxyethylstärke. In: Boldt J (Hrsg) Volumenersatztherapie. Thieme, Stuttgart New York, S 80-95

3. Schürmann M (2002) Ist das posttraumatische "complex regional pain syndrome type I" ein lokales „SIRS"? Abstract-CD, 66. Jahrestagung DGU, Berlin

4. Scola E (2003) Algodystrophie. In: Nerlich M, Berger A (Hrsg) Weichteilverletzungen und -infektionen (Tscherne Unfallchirurgie). Springer, Berlin Heidelberg New York, S 384-393

5. Scola E, Schliack H (1991) Das posttraumatische Sudeck-Syndrom. Dtsch Ärztebl 34/35A: 28032809

6. Vogel T (2002) Langzeitprognose des "complex regional pain syndrome type I". Abstract-CD, 66. Jahrestagung DGU, Berlin 